\title{
Innervation of digital joints: an anatomical overview
}

\author{
S. Gandolfi ${ }^{1} \cdot$ I. Auquit-Auckbur ${ }^{1} \cdot$ B. Chaput ${ }^{2} \cdot$ F. Duparc ${ }^{3,4}$ \\ Received: 12 February 2021 / Accepted: 16 April 2021 / Published online: 6 May 2021 \\ (c) The Author(s), under exclusive licence to Springer-Verlag France SAS, part of Springer Nature 2021
}

\begin{abstract}
Introduction The innervation of the digital joints as well as the anatomical relationships of the articular branches is present in this anatomical work to determine the technical feasibility of a selective and efficient denervation of the digital joints.

Materials and methods A study of 40 distal interphalangeal (DIP), 40 proximal interphalangeal (PIP), 50 metacarpophalangeal (MCP), 10 interphalangeal (IP) of the thumb, and 10 trapezo-metacarpophalangeal (TMC) joints was performed on ten hands. Under magnification and a proper surgical approach, we collected the course, the source origin, the number of articular nerve branches, and their caliber.

Results In total, 118 nerve branches arising from the proper palmar digital nerves were found on 10 DIP of each dissected long finger $(n=40)$. A total of 226 nerve branches were found on 10 PIPs of each long finger $(n=40)$, of which 204 branches $(90.3 \%)$ had a palmar origin. Dorsal innervation was found for the ring and little finger, originating from the dorso-ulnar digital nerve. 212 branches were found on $10 \mathrm{MCP}$ of long fingers $(n=40)$, including 87 branches of palmar origin $(41.1 \%)$, 107 branches of dorsal origin (50.4\%), and 18 branches of the motor branch of the ulnar nerve (8.5\%). 42 articular branches directed to the TMC joint $(n=10)$ were found. 13 branches $(31 \%)$ originated from the anterior sensory branch of the radial nerve, 13 branches (31\%) originated from the lateral cutaneous nerve of the forearm, 5 branches (12\%) originated from the palmar cutaneous branch of the median nerve, and $11(26 \%)$ branches originated from the thenar branch of the median nerve. The involvement of the sensory anterior branch of the radial nerve was always present for the innervation of each TMC.

Discussion and conclusion Our research shows that finger joints receive their primary innervation from small branches of the digital nerves with the exception of the MCP joint and the TMC joint. To obtain an efficient and a selective digital denervation for articular pain relief, it is necessary to plan the best surgical approach and it is crucial to recognize the articular nervous branch localization and source.
\end{abstract}

Keywords Anatomy $\cdot$ Nerve $\cdot$ Joint $\cdot$ Digit $\cdot$ Hand $\cdot$ Denervation $\cdot$ Interphalangeal $\cdot$ Metacarpophalangeal

\section{Introduction}

Surgical joint denervation is the interruption of sensory afferent articular branches to alleviating articular pain.

F. Duparc

fabrice.duparc@univ-rouen.fr

1 Department of Plastic, Reconstructive and Hand Surgery, Rouen University Hospital, Rouen, France

2 Department of Plastic and Reconstructive Surgery, Toulouse University Hospital, Toulouse, France

3 Department of Orthopedic Surgery, Rouen University Hospital, Rouen, France

4 Laboratory of Anatomy, Faculty of Medicine, Rouen University, 22 boulevard Gambetta, 76183 Rouen, France
Thanks to the improvements in anatomical knowledge, this principle was applied to the wrist by Wilhelm in 1966 [25]. This technique had a strong impact in Germany until its introduction in France by Foucher in the 90 s [10], and then developed by several authors such as Laulan and Le Nen $[14,16]$.

Whatever the denervation considered, the principle is always the same: interrupt the painful afferents by selective isolating sensory articular nerve branches, to treat the pain associated with the articular degeneration.

The indications are comparable for the different joints: degenerative or post-traumatic painful osteoarthritis when satisfactory reconstruction is not possible or when non-surgical conservative treatment is exceeded [11].

In case of osteoarthritis (OA) with preserved motion, articular denervation may be an effective alternative to 
arthroplasty or arthrodesis, to reduce or to treat a painful discomfort [11].

Hand osteoarthritis can affect different joints, mainly the distal interphalangeal joint (PID), followed by the proximal interphalangeal joint (PIP), the trapezometacarpal joint (TMC), and the metacarpophalangeal joint (MCP) [17, 24, 26].

When conservative non-surgical treatment fails to control the OA pain, patients may be offered arthrodesis or joint replacement. Both procedures have disadvantages and require a long recovery period and immobilization.

Joint denervation is a simple and effective technique who does not interfere with articular biomechanics, and it is generally consider a mini-invasive treatment $[10,11,14,16]$.

Despite being well used for the wrist and the carpus, finger joint denervation is still underestimated and surgical techniques are still very little codified. Indeed, these surgical procedures are considered technically difficult, because only few nervous branches are specifically isolated: a detailed knowledge of the anatomy of the articular nerves is therefore essential.

While the articular anatomy of digital joints is known in detail, their innervation is still debated [3, 6-8, 12, 15].

Cozzi [7] and Lorea et al. [19] have already pointed it out: the precise knowledge of the anatomy of innervation of the digital joints is an essential prerequisite for the description of a denervation technique.

The aim of this anatomical work is to describe the innervation of the digital joints as well as the anatomical relationships of the articular branches to determine the technical feasibility of a selective and efficient denervation of the digital joints in case of painful arthrosis.

\section{Materials and methods}

In the Anatomical Laboratory of the Faculty of Medicine of Rouen-Normandy University, France, a dissection of ten hands (five right hands, five left hands) corresponding to five donated bodies to science (three men, two women) was performed. All the procedures were done in full respect to the Donation Program Rules of the Rouen-Normandy University.

No apparent scars or deformities of the fingers were found in any body.

A study of 40 distal interphalangeal (DIP), 40 proximal interphalangeal (PIP), 50 metacarpophalangeal (MCP), 10 interphalangeal (IP) of the thumb, and 10 trapezo-metacarpophalangeal (TMC) joints was performed.

Each dissection was carried out on fresh hands, using magnifying surgical loupes (Prismatic Loupes, Keeler, UK) X 3.5 and X 5.5, proceeding from the proximal to the distal.
All the dissected nervous branches implanting on the joint capsules were considered to participate on the innervation of the digital joints.

In all cases, the course, the source origin, and the number of articular nerve branches were recorded and the caliber of the nerve branches was measured at the origin through a micrometer caliper (Mitutuyo ${ }^{\mathrm{R}}$ System).

\section{Surgical approach}

Exposure of the DIP joint was performed using a dorsal approach by elevating a U-shaped flap with a proximal base extending from the eponychial fold to the joint according to the method used by Arenas-Prat [1] during DIPs' denervations.

Once the articular branches isolated, the dissection was continued in the dorso-palmar direction and distally proximally to find the proper palmar digital nerves sources.

The innervation of the PIP joint was studied through a longitudinal palmar and dorsal approach. The proper palmar digital nerves were exposed through the palmar approach (Fig. 1), allowing visualization of the articular nervous contingencies.

A dorsal longitudinal incision was realized from the MCP joint to the head of the distal phalange, exposing the extensor apparatus of the fingers and allowing the visualization of the articular nervous contingencies originating from the dorsal nerves (Fig. 2).

Exposure of the articular nerves to the MCP joint was achieved through a mixed palmar and dorsal approach. A single incision at the digito-palmar fold completed by a dissection of a palmar skin flap elevated from the radial side to the ulnar side allowed simultaneous exposure of the 4 MCPs of the long fingers to isolate the articular nerves from the palmar digital nerves (Fig. 3).

Once this step completed, the flexor system was lifted to follow the articular nerve branches for MCP originating from the motor branch of the ulnar nerve (Fig. 4).

A transversal dorsal incision at the distal phalange base level allowed a proximo-distal detachment of a dorsal skin flap, revealing the dorsal digital nerves, source of the dorsal articular nerves at the MCP of the four long fingers (Fig. 5).

The articular nerves to the thumb were studied through a palmar approach for the MCP (Fig. 6), and a mixed dorsal and palmar approach for the IP, according to the method described for the PIPs.

For the TMC joint, the nerves involved in dissections were: the median nerve, with its thenar and palmar cutaneous branches, branches from the anterior sensory branches of the radial nerve, and branches from the lateral cutaneous nerve of the forearm.

These nerves were isolated in the distal third of the forearm, and then dissected from proximal to distal until their terminal 




Fig. 1 Palmar surgical approach of the PIP joint of the index (left side) through a longitudinal palmar incision. Note the articular nerve branch separating from the radial proper palmar digital nerve under the blue square. 1: proper palmar digital nerve; 2: articular nerve branch

arborization, with identification of the articular branches intended for TMC (Figs. 7 and 8).

At the palmar level, dissection of the median nerve and its thenar and palmar skin branches was realized after excision of the skin tissue of the palm and thenar eminence, and after opening the carpal tunnel (Figs. 9 and 10).

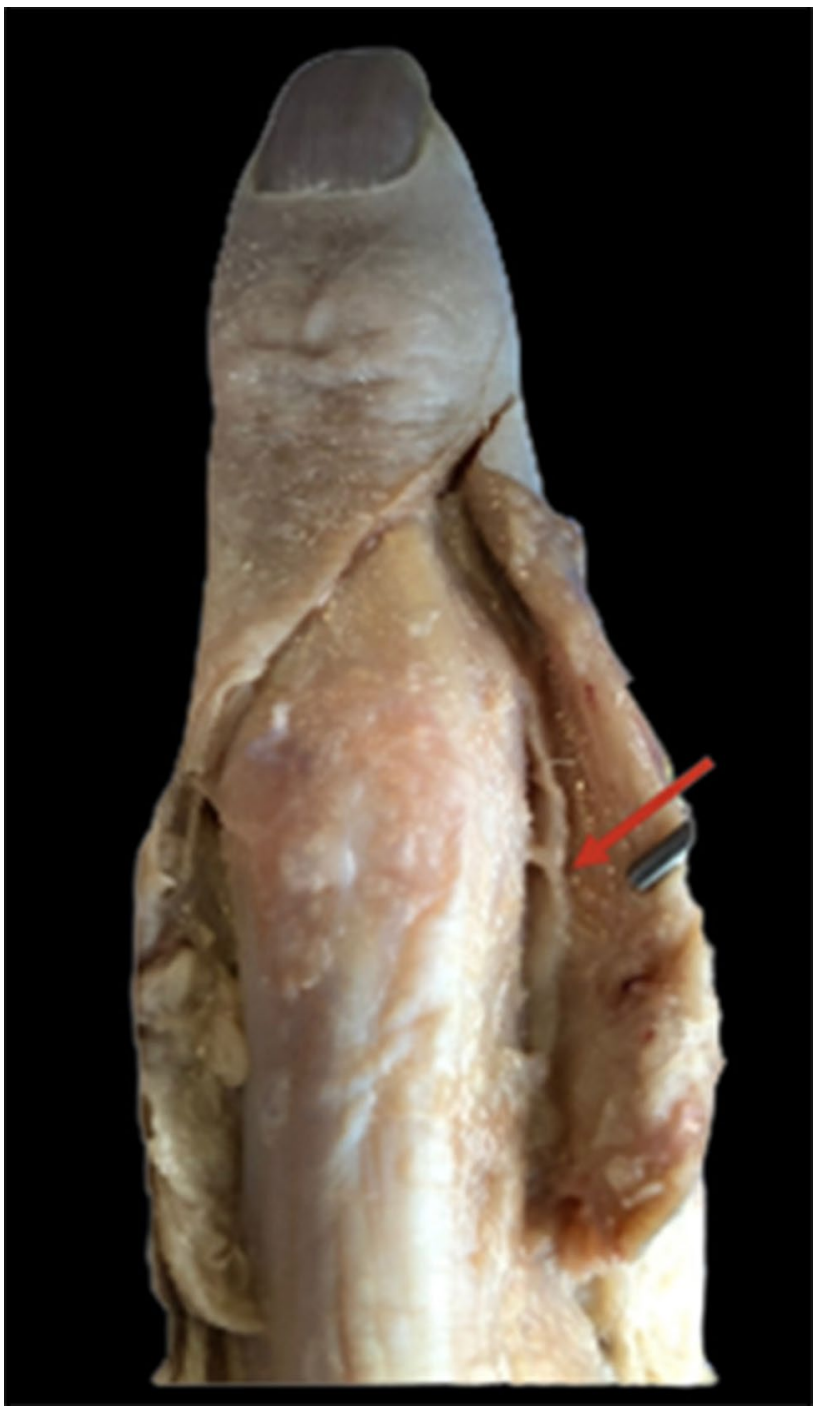

Fig. 2 Dorsal surgical approach the PIP of the medium (right side) through a dorsal longitudinal incision. The red arrow shows a dorsal digital ulnar articular branch

\section{Results}

\section{Results from the DIP joint innervation}

In all cases, the articular nerve branches originated from the proper palmar digital nerve, from the main trunk or from the trifurcation. From 0 to 3 articular branches were detached from the source branch and proceeded directly towards the articular capsule.

In total, 118 nerve branches were found on $10 \mathrm{DIP}$ of each dissected long finger $(n=40) .72$ branches $(61 \%)$ detached from the main trunk and 46 branches (39\%) detached from the trifurcation.

The average distance, measured at the base of the source branch, was $1.5 \mathrm{~mm}(\min 0.7, \max 2.3)$. 

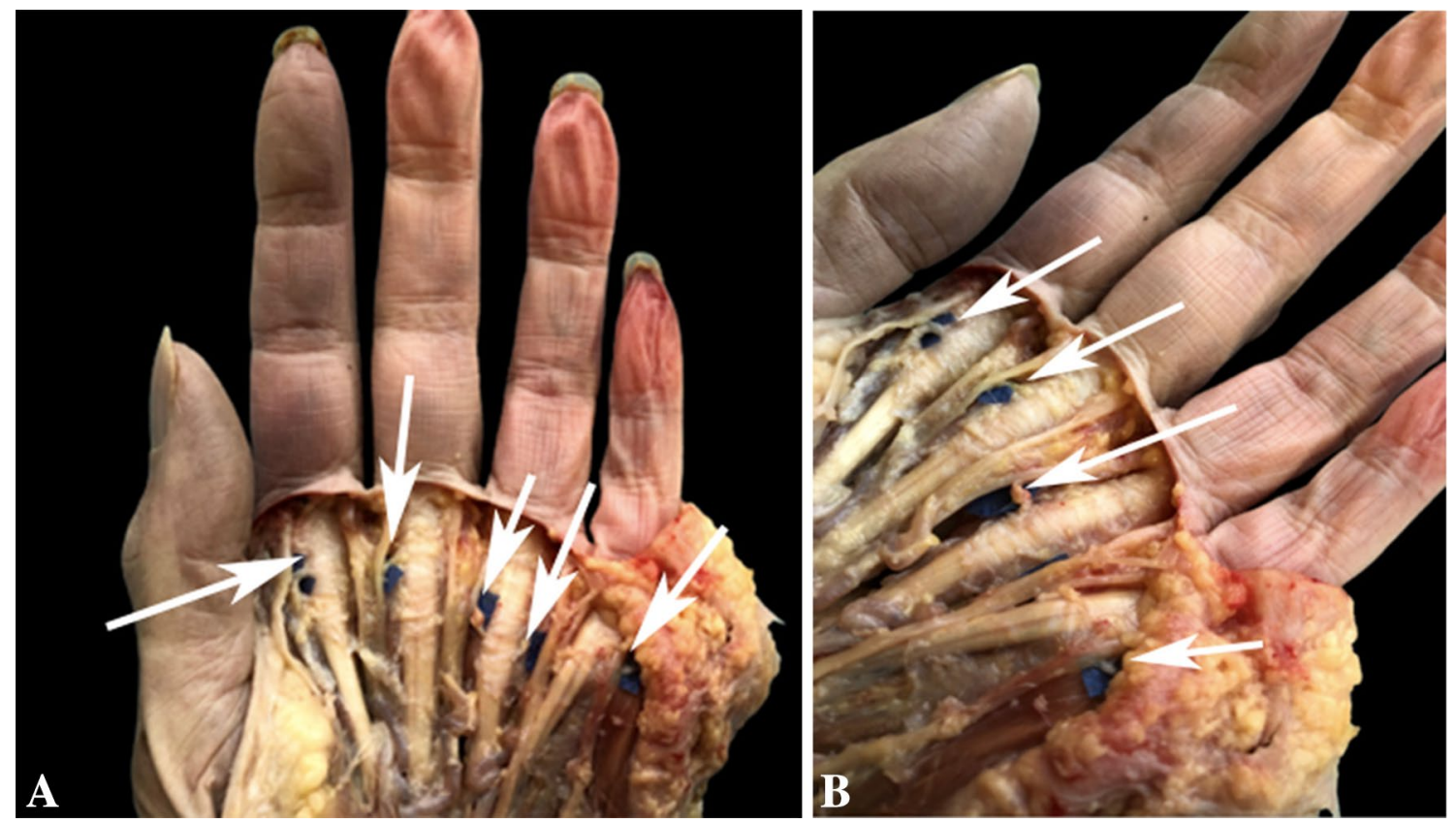

Fig. 3 a, b Palmar approach to highlight the articular branches of the MCP joints from the proper palmar digital nerves isolated on the blue squares (White arrows). Left hand



Fig. 4 MCP joint nerve contingencies from the deep branch of the ulnar nerve after flexor apparatus resection highlighted under the blue square. Left hand

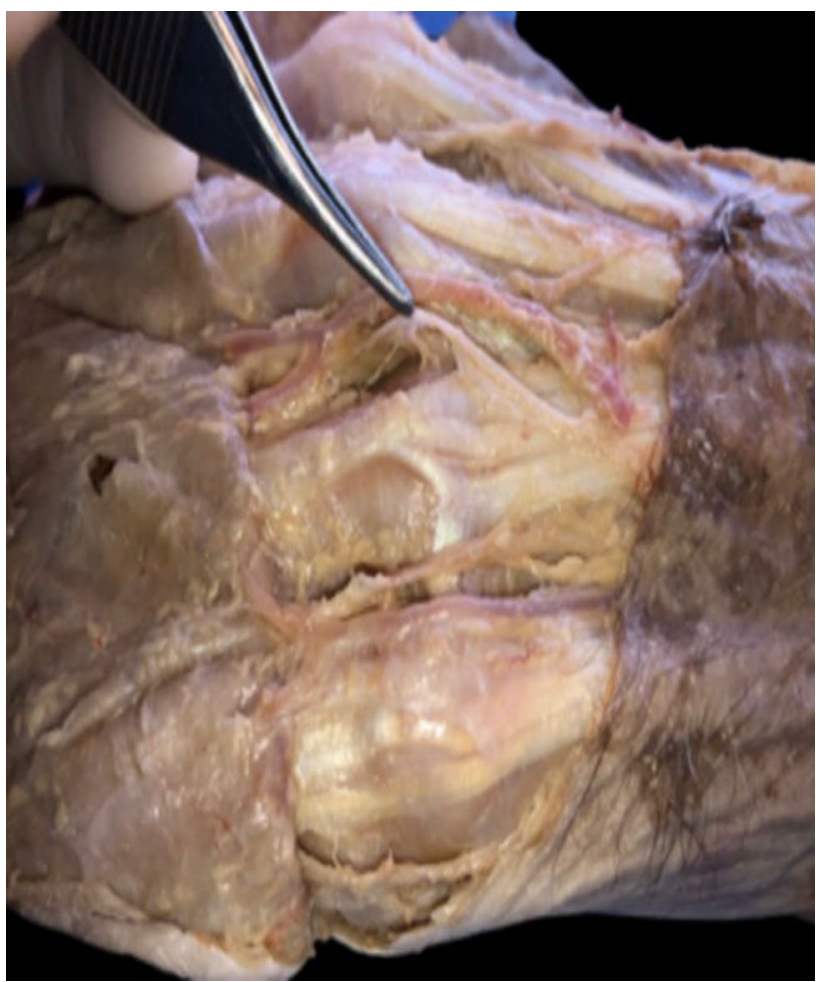

Fig. 5 Dorsal approach of MCP joints showing the dorsal contingency of the articular nerves. Note under the forceps the dorsal radial digital nerve of the second MCP joint of a right hand 




Fig. 6 Palmar approach of the first MCP showing a palmar nerve branch originating from the proper radial palmar digital nerve highlighted under the blue square. Left hand

The results of the number of branches, as well as their average size are summarized in Table 1.

No branches from the dorsal digital nerves were found.

\section{Results from the PIP joint innervation}

In all the dissections, the PIP joint was innervated by nerve branches from the proper palmar digital nerves (from 2 to 4 branches). Dorsal innervation was only found for the ring and little finger, originating from the dorso-ulnar digital nerve.

A total of 226 nerve branches were found on 10 PIPs of each long finger $(n=40)$, of which 204 branches $(90.3 \%)$ had a palmar origin.

Dorsally, no joint branch was found at the level of the index finger and middle finger. However, from 0 to 3 branches (mean caliber 1.24 ; min 0.84 , max 1.32 , $\mathrm{SD} \pm 0.08$ ), originating from the dorso-ulnar sensory nerve were found on the ulnar side of the PIP of the ring and little finger for a total of 22 branches $(9.7 \%)$.

The articular nerves from the proper palmar digital nerves entered the space between the flexor tendon sheath and the head of the proximal phalanx (P1) and divided into branches that supplied the volar part of the joint capsule, the head of
$\mathrm{P} 1$, the tendon sheath, and the lateral ligaments of the joint capsule.

The dorsal articular nerves originated from the dorsal branches of the ulnar nerve. These nerves entered the space between the medial band of the extensor apparatus and the lateral band and arborized in the dorsal capsule.

The overall average distance between two articular branches at the base was $5 \mathrm{~mm}(\min 3$; $\max 8)$.

The results of the number of palmar branches found, as well as their average size are summarized in Table 2.

\section{Results from the PI joint innervation of the thumb}

In all dissections, the PI joint was innervated by nerve branches originated from the proper palmar digital nerves.

Dorsal innervation was not found.

In total, 26 articular nerve branches destined for the IP of the thumb were explored. In all cases, one branch arising from the radial proper palmar digital nerve detached from the main trunk to join the joint capsule of the PI in its anterior-external side.

In six cases, the ulnar proper palmar digital nerve gave off two branches to IP capsule in its anterior-internal side.

The average caliber of the branches was $1.57 \mathrm{~mm}$ (min 1.49; $\max 1.61 ; \mathrm{SD} \pm 0.08$ ) for the radial side and $1.52 \mathrm{~mm}$ $(\min 1.37 ; \max 1.58 ; \mathrm{SD} \pm 0.07)$ for the ulnar side.

The average distance between two articular branches at the base of the articular capsule for the ulnar side was 1.8 $\mathrm{mm}(\min 1 ; \max 2.1)$.

\section{Results from the MCP joint innervation}

MCP joints of the four last fingers receive articular branches from the palmar digital nerves, the dorsal digital nerves, and the motor branch of the ulnar nerve.

In all the joints, from 0 to 2 branches from the palmar digital nerves and from 0 to 3 branches from the dorsal digital nerves were found.

However, the nervous contingency from the motor branch of the ulnar nerve was found more rarely: no branch was found on the radial side of each of the $10 \mathrm{MCP}$ of the index and little finger.

In total, 212 branches were found on $10 \mathrm{MCP}$ of long fingers $(n=40)$, including 87 branches of palmar origin (41.1\%), 107 branches of dorsal origin $(50.4 \%)$, and 18 branches of the motor branch of the ulnar nerve (8.5\%).

The articular nerves which originated from the radial and ulnar sides of the palmar digital nerves (0-2 branches), systematically attached to the joint capsule, the palmar plate and the flexor sheath, the sagittal band of the extensor apparatus, and the dorsal part of the metacarpal head.

The deep branch of the ulnar nerve, deeply located under the flexor apparatus, gave off from 0 to 1 articular 

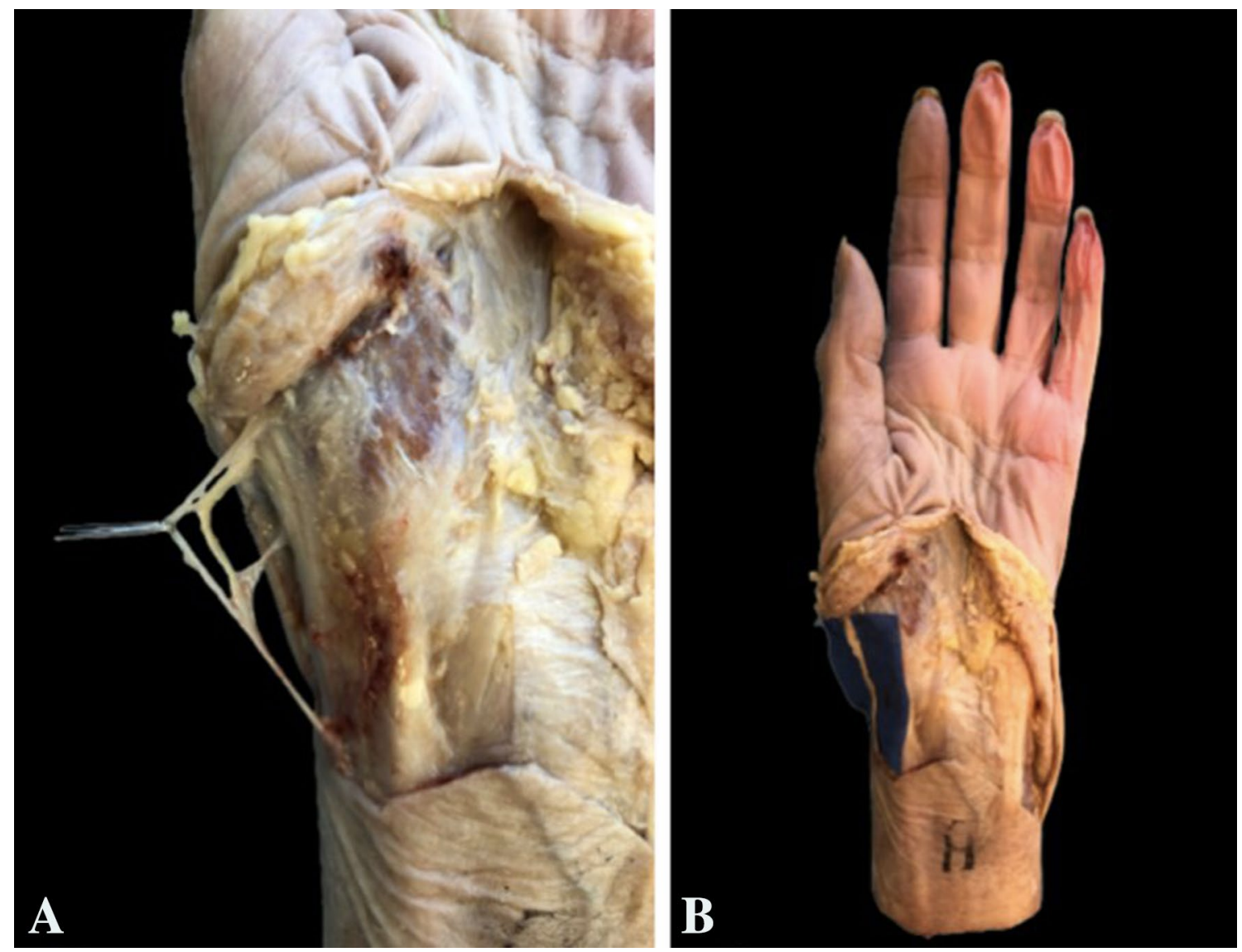

Fig. 7 Visualization under the forceps of the articular branches for TMC (a) from the lateral cutaneous nerve of the forearm (b) highlighted under the blue square. Left hand

branch ramifying proximally to the A1 pulley, inserting the palmar and lateral side of the articular capsule, at the level of the metacarpal head, on the flexor sheath, and on the lateral ligaments of the MCP joint.

For the branches coming from the dorsal and palmar digital nerves, the mean distance between two articular branches at the base was $6 \mathrm{~mm}(\min 4$; $\max 9)$.

In no case, there was more than one branch penetrating the MCP capsule from the deep ulnar nerve.

Tables 3, 4, and 5 summarize the various data on the innervation of MCP in long fingers, respectively, from the palmar, dorsal, and from the motor branch of the ulnar nerve.

For the 1st MCP, no nervous contingency from the dorsal nerves and from the motor branch of the ulnar nerve was found.

In all cases, from 1 to 2 nerve branches from the palmar digital nerves were found on every $10 \mathrm{MCP}$ of the thumbs, for a total of 16 nerve branches found. These branches had a direct course towards the anterior-external and anteriorinternal side of the joint capsule.
The average caliber of the branches was $1.61 \mathrm{~mm}$ (min 1.59 ; $\max 1.62 ; \mathrm{SD} \pm 0.02$ ) for the radial side and $1.38 \mathrm{~mm}$ (min 1.32; $\max 1.43 ; \mathrm{SD} \pm 0.04$ ) for the ulnar side.

The overall average distance between two articular branches at the base of the joint capsule was $3 \mathrm{~mm}$ (min 1; $\max 4$ ).

\section{Results from the innervation of the TMC joint}

In total, 42 articular branches directed to the TMC joint $(n=10)$ were found.

13 branches $(31 \%)$ originated from the anterior sensory branch of the radial nerve, 13 branches $(31 \%)$ originated from the lateral cutaneous nerve of the forearm, 5 branches $(12 \%)$ originated from the palmar cutaneous branch of the median nerve, and 11 (26\%) branches originated from the thenar branch of the median nerve.

The involvement of the sensory anterior branch of the radial nerve was always present for the innervation of each TMC. Articular nerve branches detached from the most external branch of the trifurcation. These branches 

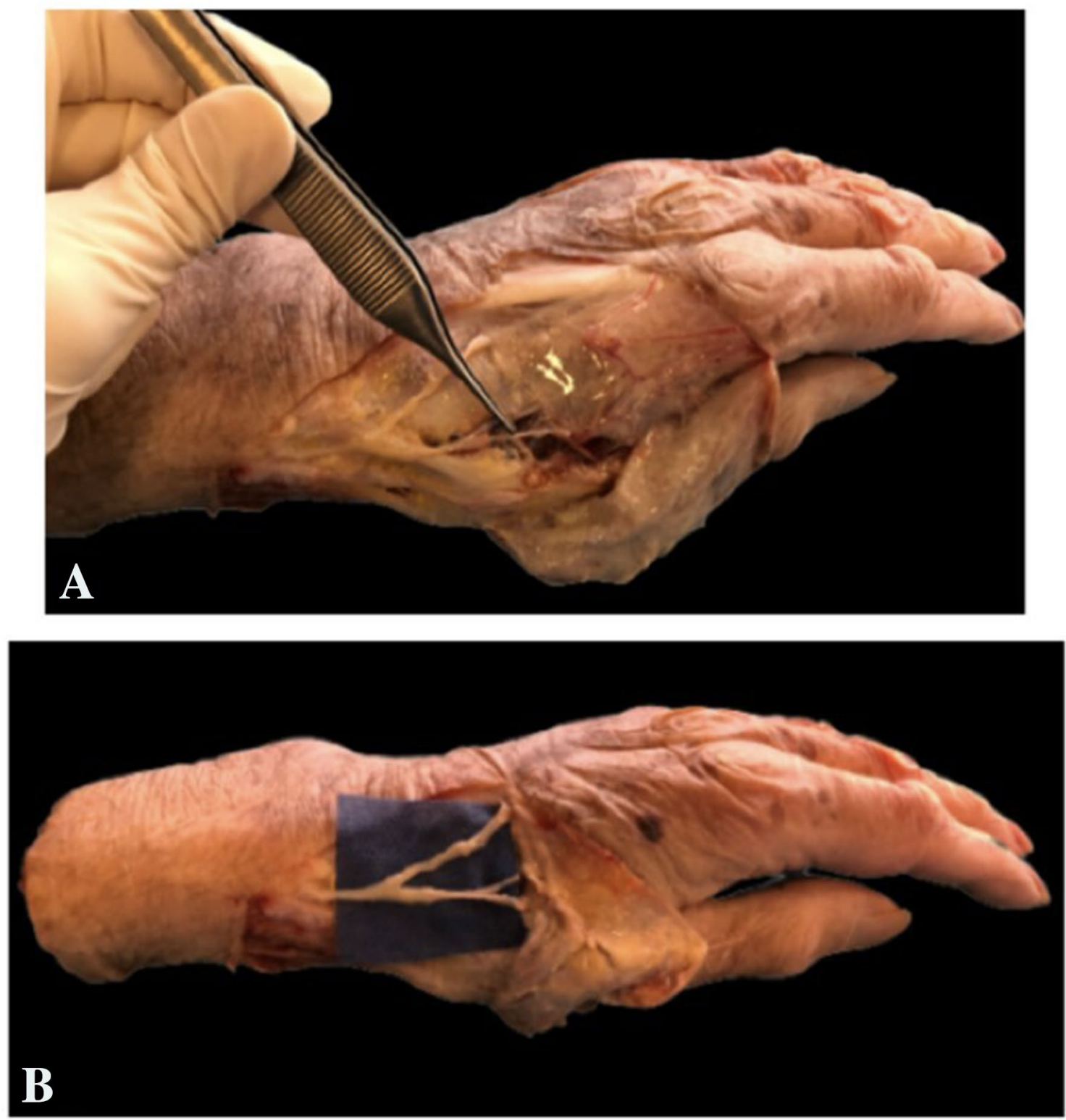

Fig. 8 Visualization under the forceps of the articular branches for TMC (a) from the sensory anterior branches of the radial nerve (b) highlighted under the blue square. Left hand

originated in the region of entered the joint capsule of the TMC dorsally, passing between the extensor digitorum brevis and extensor longus of the thumb. In all cases, there were from 1 to 3 branches with an average caliber all branches combined of $1.62 \mathrm{~mm}(\min 1.21$; $\max 1.87$; $\mathrm{SD} \pm 0.05)$.

For the lateral cutaneous nerve of the forearm, the articular branches for TMC in all cases detached from the most volar branch, running parallel to the radial artery, and then entered the anti-brachial fascia before terminal division under the first compartment of the extensors before reaching the TMC.
These branches were found in nine cases out of ten. The mean caliber was $1.41 \mathrm{~mm}(\min 1.35 ; \max 1.43 ; \mathrm{SD} \pm 0.09)$.

Median nerve involvement in TMC innervation was found in all dissections: five out of ten for the palmar cutaneous branch and nine out of ten for the thenar branch. In four cases, there were both palmar cutaneous branches and thenar branches.

The palmar cutaneous branch originated $3-5 \mathrm{~cm}$ above the radial styloid (mean $4 \mathrm{~cm}$ ). Its articular branches crossed the annular ligament of the carpus and entered the anterior aspect of the TMC above the trapezius. 



Fig. 9 Visualization under the forceps of the palmar cutaneous branch of the median nerve for TMC. Left hand

The mean caliber for the palmar cutaneous branches was $1.37 \mathrm{~mm}(\min 1.25 ; \max 1.44 ; \mathrm{SD} \pm 0.08)$.

In six cases of nine (67\%), the articular branches originated from the thenar branch had an extra-ligamentous path, left the carpal tunnel with an anterior-superior run before entering the articular capsule of the TMC at the level of the proximal insertions of the opponent muscle. In the remaining three cases (33\%), the course of the thenar branch was sub-ligamentous. The mean caliber for the thenar articular branches was $1.21 \mathrm{~mm}$ (min 1.09; $\max 1.24$; $\mathrm{SD} \pm 0.04)$.

Table 6 summarizes the modalities of nerves involvement in $\mathrm{TMC}$ joint.

\section{Discussion}

Our research shows that finger joints receive their primary innervation from small branches of the digital nerves with the exception for the MCP and the TMC joint.

The DIP joint receives a network of very tiny nervous branches arising from palmar digital nerves, either from main trunk or from the trifurcation and directly penetrate in the joint capsule. Although the distal interphalangeal joint is the most frequent site of osteoarthritis in the hand, very few anatomical works has been done on this joint looking at its innervation $[12,13]$. Unlike these studies, we did not find any branches arising from dorsal digital nerves for the DIP joint, maybe for the little caliber of these branches. However, if a surgical procedure of a DIP denervation has to be planned with a U-shaped proximal dorsal flap as Arenas-Prat described [1]. Articular dorsal branches could be not necessarily identified, because the elevation of a dorsal flap along the DIP joint line will potentially englobe dorsal articular branches, detaching them form the articular capsule. At contrary, nervous branchlets from the palmar digital nerves have to be sufficiently selected at the origin. In our work, the average caliber of these branches at the origin is 0.61 $\mathrm{mm}$, which is very difficult to individualize. It is therefore necessary to have a knowledge of the localization of these branches, to isolate the proper palmar digital nerve at the level of the DIP joint capsule.

PIPs joints have a mixed innervation, from palmar and from dorsal digital nerves. Palmar branches detached from the main trunk about 8-17 $\mathrm{mm} \mathrm{[20],} \mathrm{from} \mathrm{the} \mathrm{joint} \mathrm{line} \mathrm{and}$ directly reach the joint capsule and the head on the proximal phalanx with an emergency angle of $20^{\circ}-45^{\circ}$ [20], passing 
Fig. 10 Visualization under the blue square of the thenar branch of the median nerve for TMC. Left hand

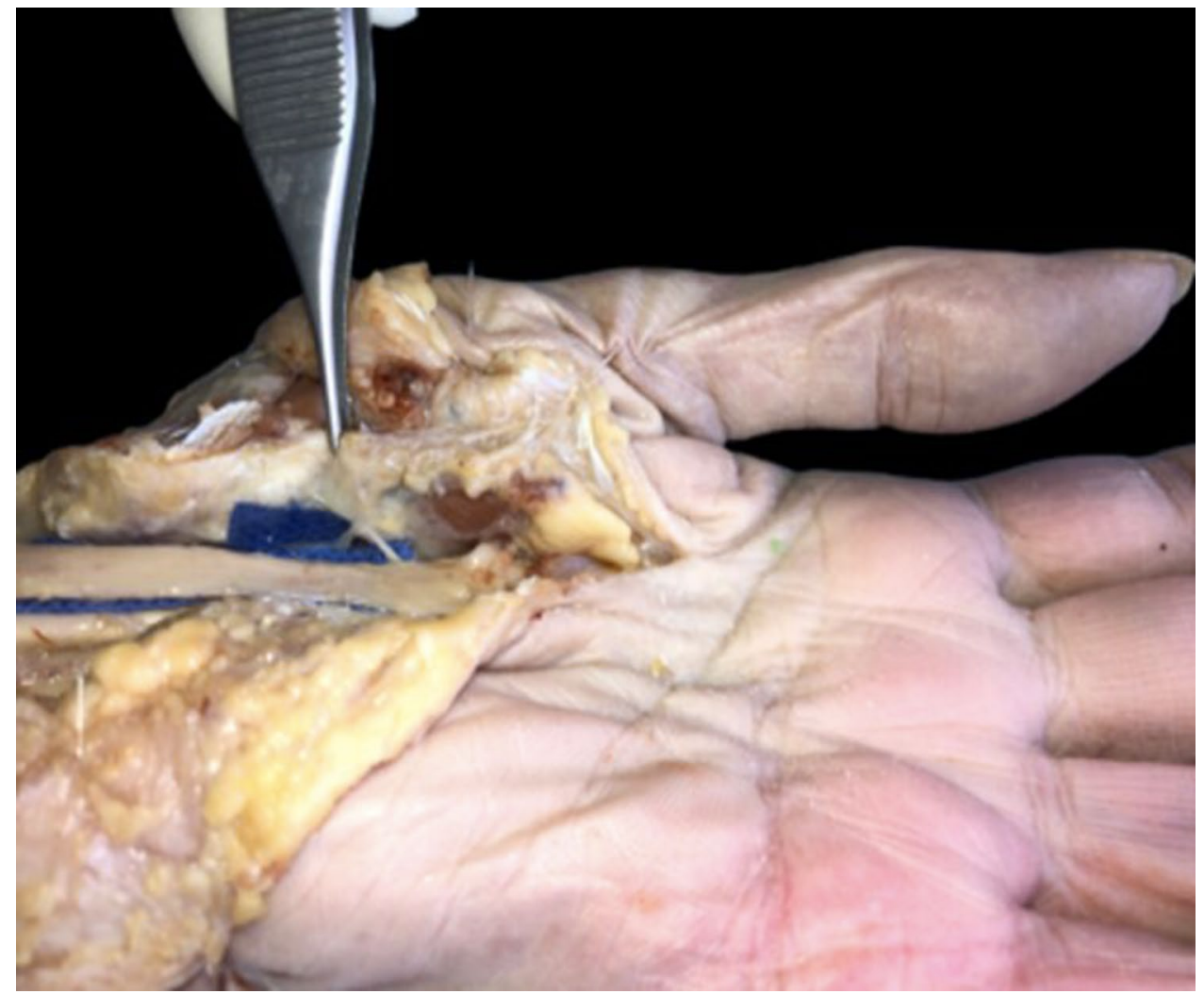

\begin{tabular}{llllll}
\hline & \multicolumn{2}{l}{ R-DIP } & & U-DIP & \\
\cline { 2 - 3 } & Branches $(n)$ & Caliber $(\mathrm{mm})$ & & Branches $(n)$ & Caliber $(\mathrm{mm})$ \\
\hline Index $(n=10)$ & 18 & $0.66 \pm 0.07$ & & 14 & $0.63 \pm 0.08$ \\
Middle finger $(n=10)$ & 17 & $0.63 \pm 0.08$ & & 13 & $0.61 \pm 0.07$ \\
Ring finger $(n=10)$ & 14 & $0.58 \pm 0.08$ & & 15 & $0.68 \pm 0.08$ \\
Little finger $(n=10)$ & 13 & $0.52 \pm 0.07$ & & 14 & $0.59 \pm 0.09$ \\
\hline
\end{tabular}

$R$-DIP radial side of the DIP; $U$-DIP ulnar side of the DIP; Branches total number of branches; Caliber mean caliber with standard deviation

\begin{tabular}{llllll}
\hline & \multicolumn{2}{l}{ R-PIP } & & U-PIP & \\
\cline { 2 - 3 } & Branches $(n)$ & Caliber $(\mathrm{mm})$ & & Branches $(n)$ & Caliber $(\mathrm{mm})$ \\
\hline Index $(n=10)$ & 23 & $1.87 \pm 0.06$ & & 21 & $1.70 \pm 0.07$ \\
Middle finger $(n=10)$ & 24 & $1.54 \pm 0.09$ & & 23 & $1.31 \pm 0.07$ \\
Ring finger $(n=10)$ & 24 & $1.22 \pm 0.08$ & & 26 & $1.72 \pm 0.08$ \\
Little finger $(n=10)$ & 31 & $0.68 \pm 0.06$ & & 32 & $0.79 \pm 0.07$ \\
\hline
\end{tabular}

$R$-PIP radial side of the PIP; U-PIP ulnar side of the PIP; Branches total number of branches; Caliber mean caliber with standard deviation the space between the flexor tendon sheath and the proximal phalanx. Inconstant dorsal articular nerves entered the space between the medial band of the extensor apparatus and the lateral band and then arborized in the dorsal capsule. For PIP joints, our results differ from literature. In contrast of
Schultz [22] and Braga-Silva [4] who did not find any dorsal contingencies, we found that PIPs are innervated from palmar and dorsal proper digital nerves. These data suggest that to realize an efficient denervation of the PIP joint, a palmar surgical approach could not be sufficient. A mixed 
Table 3 Results from MCP joint volar innervation

\begin{tabular}{llllll}
\hline & \multicolumn{2}{l}{ VR-MCP } & & \multicolumn{2}{l}{ VU-MCP } \\
\cline { 2 - 3 } & Branches $(n)$ & Caliber $(\mathrm{mm})$ & & Branches $(n)$ & Caliber $(\mathrm{mm})$ \\
\hline Index $(n=10)$ & 15 & $1.94 \pm 0.07$ & & 14 & $1.64 \pm 0.08$ \\
Middle finger $(n=10)$ & 12 & $1.88 \pm 0.06$ & & 13 & $1.58 \pm 0.07$ \\
Ring finger $(n=10)$ & 5 & $1.63 \pm 0.07$ & & 6 & $1.66 \pm 0.08$ \\
Little finger $(n=10)$ & 8 & $1.58 \pm 0.09$ & & 14 & $1.49 \pm 0.09$ \\
\hline
\end{tabular}

$V R-M C P$ volar and radial side of the MCP; $V U-M C P$ volar and ulnar side of the MCP; Branches total number of branches; Caliber mean caliber with standard deviation

\begin{tabular}{llllll}
\hline & \multicolumn{2}{l}{ DR-MCP } & & \multicolumn{2}{l}{ DU-MCP } \\
\cline { 2 - 3 } & Branches $(n)$ & Caliber $(\mathrm{mm})$ & & Branches $(n)$ & Caliber $(\mathrm{mm})$ \\
\hline Index $(n=10)$ & 18 & $1.54 \pm 0.08$ & & 12 & $1.22 \pm 0,07$ \\
Middle finger $(n=10)$ & 15 & $1.46 \pm 0.07$ & & 12 & $1.23 \pm 0.07$ \\
Ring finger $(n=10)$ & 11 & $1.32 \pm 0.06$ & & 18 & $1.37 \pm 0.08$ \\
Little finger $(n=10)$ & 8 & $1.15 \pm 0.07$ & & 13 & $1.42 \pm 0.07$ \\
\hline
\end{tabular}

$D R-M C P$ dorso-radial side of the MCP; $D U-M C P$ dorso-ulnar side of the MCP; Branches total number of branches; Caliber mean caliber with standard deviation

\begin{tabular}{llllll}
\hline & \multicolumn{2}{l}{ RDUN-MCP } & & \multicolumn{2}{l}{ UDUN-MCP } \\
& Branches $(n)$ & Caliber $(\mathrm{mm})$ & & Branches $(n)$ & Caliber $(\mathrm{mm})$ \\
\hline Index $(n=10)$ & N.F & N.F & & 7 & $1.41 \pm 0.02$ \\
Middle finger $(n=10)$ & 5 & $1.18 \pm 0.04$ & & 1 & $1.21 \pm 0.05$ \\
Ring finger $(n=10)$ & 3 & $1.12 \pm 0.11$ & & 2 & $1.12 \pm 0.09$ \\
Little finger $(n=10)$ & N.F & N.F & & 8 & $1.09 \pm 0.1$ \\
\hline
\end{tabular}

$R D U N-M C P$ radial side of the MCP from the deep ulnar nerve; UDUN-MCP ulnar side of the MCP from the deep ulnar nerve; Branches total number of branches; Caliber mean caliber with standard deviation; N.F. not found
Table 5 Results from MCP joint innervation from the deep ulnar nerve
Table 4 Results from MCP joint dorsal innervation
Table 6 Modalities of nerves involvement in TMC joint, with the total number of branches arising from the main trunks, the number of times $(t)$ when articular branches were found, and their caliber (mean and standard deviation)

\begin{tabular}{|c|c|c|c|c|c|}
\hline & \multirow{2}{*}{$\begin{array}{l}\text { Total } \\
\text { branches } \\
(n)\end{array}$} & \multicolumn{3}{|c|}{ Articular branches $(t)$} & \multirow{2}{*}{$\begin{array}{l}\text { Caliber } \\
(\mathrm{mm})\end{array}$} \\
\hline & & 1 branch & 2 branches & 3 branches & \\
\hline ABRN & 10 & 3 & 5 & 2 & $1.62 \pm 0.05$ \\
\hline LCFN & 9 & 6 & 2 & 1 & $1.41 \pm 0.02$ \\
\hline CPBMN & 5 & 5 & I & / & $1.37 \pm 0.08$ \\
\hline TBMN & 9 & 7 & 2 & / & $1.21 \pm 0.04$ \\
\hline
\end{tabular}

$A B R N$ anterior branch of the radial nerve; $L C F N$ lateral cutaneous nerve of the forearm; $C P B M N$ cutaneous palmar branch of the median nerve; $T B M N$ thenar branch of the median nerve

palmar-dorsal approach or a bilateral mid-lateral approach exposing both sides of the PIP joint are necessary to fully select palmar and dorsal nervous contingencies. Our results from the origin of nerve branches for PIP joint are supported by the Gray and Gardner [20] fetal study with silver staining, who found also a participation of dorsal nerves. Pastrana [20], also found in his anatomic study a dorsal contingency but only for the little finger. We found dorsal branches for the fourth and the fifth finger. According to Pastrana [20], we consider these branches as the terminal branches of the dorso-ulnar sensory nerve.

For PI joint of the thumb, we found some direct branches to the capsule originated from the proper palmar digital nerves. We did not find any dorsal branches, and lacks of data in the literature about the innervation of this joint could permit to state an only palmar contingency, given the relative robust caliber of articular nervous branches at PI joint level (average $1.54 \mathrm{~mm}$ ), could supply the entire innervation of this articulation.

MCP joints are innervated by branches arising from each palmar digital nerve, from dorsal digital nerves and from one volar branch detaching from the deep motor branch of 
the ulnar nerve. For these joints, our results do not differ from literature $[5,13]$, excepting that we did not observed any deep ulnar contingency on the radial side of the index and of the little finger.

Dissection of these branches was quite laborious, because it is necessary to recline the flexor apparatus to have access. A surgical mixed approach, palmar and dorsal, is therefore required to select deep ulnar nerve branches directed to the MCP joints.

Our results confirmed that the radial nerve, the lateral cutaneous nerve of the forearm, and the median nerve participate to the innervation of the TMC joints. Our results differed from those published by Cozzi [7], in his extensive anatomic study of 500 hands. The participation of the dorsal sensory branch of the radial nerve, although found in all our dissections, does not play an exclusive role in the innervation of the TMC joints. Our results agreed more with the more recent Pupon [21] and Loréa's studies [18, 19] where the participation of the three main nervous trunks is present, and the median nerve has a much more substantial participation than suggested by Cozzi in his study [7]. He mentions a participation of the thenar branch and of the palmar cutaneous branch only in $5 \%$ of cases, whereas we found a median nerve involvement in all dissections: five out of ten cases for the palmar cutaneous branch and nine out of ten cases for the thenar branch. In four cases, there were both palmar cutaneous branches and thenar branches.

The discrepancies observed between our results and those of the literature can be explained by a different approach to the dissections: a predominant innervation by the radial nerve was confirmed when an only dorsal surgical approached to the TMC joint was planned. In this sense, to undergone an efficient surgical denervation of the TMC joint, a mixed approached as recommended by Arenas-Prat in his Wagner approach [2] seems to us more suitable. It is about to offer a wider access to the TMC joint in its dorsal and volar aspect including the trapezium and metacarpal bases, ensuring complete dissection of all sensory branches. The Wagner approach $[2,9,23]$ requires elevation of the thenar muscles and a circumferential "degloving" above the joint capsule, fully reaching branches from the lateral cutaneous nerve of the forearm and the median nerve, including the thenar branch and the palmar cutaneous branch.

\section{Limitations of the study}

This study had some limitations. First, the number of specimens was small, but it was sufficient to characterize innervation patterns. Second, a histological analysis with selective staining for nervous tissue of the capsule might refine the anatomical description, but we found some difficulties to access laboratories during this period of COVID-19 pandemic.

\section{Conclusion}

To realize finger joint denervation, it is crucial to understand the innervation of digital joints.

Although these descriptions provide an overview of the innervation of the finger joints, it must be remembered that the caliber of articular branches could render their identification difficult intra-operatively. Therefore, it is necessary to have the knowledge of articular nervous branch localization to sufficiently perform a selective digital denervation with the best surgical approach.

Acknowledgements The authors are thankful to DR Jordane Mouton, $\mathrm{MD}$, for his precious advices, and to Mr. Bruno Belloncle for his technical assistance. The authors sincerely thank those who donated their bodies to science, so that anatomical research could be performed. Results from such research can potentially increase mankind's overall knowledge that can then improve patient care. Therefore, these donors and their families deserve our highest gratitude.

Author contributions SG: technical study, acquisition of data, and manuscript editing. IA-A: concept and design of the study. BC: manuscript editing. FD: technical study and manuscript editing.

\section{References}

1. Arenas-Prat JM (2012) Denervation of the distal interphalangeal joint. Tech Hand Surg 16:12-13

2. Arenas-Prat JM (2012) Wagner approach for first carpometacarpal joint denervation. Tech Hand Surg 16:107-109

3. Bas H, Kleinert J (1999) Anatomic variations in sensory innervation of the hand and digits. J Hand Surg 24(6):1171-1184

4. Braga-Silva J, Calcagnotto $G$ (2001) The innervation of the proximal interphalangeal joint and its application in neurectomy. J Hand Surg Br 26(6):541-543

5. Chen Y-G, McClinton MA, DaSilva MF et al (2000) Innervation of the metacarpophalangeal and interphalangeal joints: a microanatomic and histologic study of nerve endings. J Hand Sur 25A(1):128-133

6. Chikenji T, Suzuki D, Fujimiya M, Moriya T, Tsubota S (2010) Distribution of the nerve endings in the human proximal interphalangeal joint and surrounding structures. J Hand Surg Am 35(8):1286-1293

7. Cozzi EP (1991) Dénervation des articulations du poignet et de la main. In: Tubiana R (ed) Traité de chirurgie de la main, Tome IV. Editions Masson, Paris, pp 781-787

8. Dubert T, Oberlin C, Alnot JY (1990) Anatomie des nerfs articulaires du poignet: application à la technique de dénervation. Ann Chir Main Memb Super 9:15-21

9. Ehrl D, Erne HC, Broer PN, Metz C, Falter E (2016) Outcomes of denervation, joint lavage and capsular imbrication for painful thumb carpometacarpal joint osteoarthritis. J Hand Surg Eur 41(9):904-909

10. Foucher G, Long PP, Erhard L (1998) Joint denervation, a simple response to complex problems in hand surgery. Chirurgie 123:183-188

11. Gandolfi S, Carloni R, Mouton J, Auquit-Auckbur I (2020) Finger joint denervation in hand osteoarthritis: indications, surgical 
techniques and outcomes. A systematic review of published cases. Hand Surg Rehabil 39(4):239-250

12. Gray D, Gardner E (1965) The innervation of the joints of the wrist and hand. Anat Rec 151(3):261-266

13. Kim YS (2001) Innervation of metacarpophalangeal joint and distal interphalangeal joint: an anatomical and histological study. Kaibogaku Zasshi 76:313-322

14. Laulan J, Marteau E, Bacle G (2015) Wrist osteoarthritis. Orthop Traumatol Surg Res 101(1):S1-S9

15. Laroy V, Spaans F, Reulen J (1998) The sensory innervation pattern of the fingers. J Neurol 245(5):294-298

16. Le Nen D, Laulan J et al (2011) The arthritic wrist. I-the degenerative wrist: surgical treatment approaches. Orthop Traumatol Surg Res 97(4 Suppl):S31-S36. https://doi.org/10.1016/j.otsr. 2011.03.006

17. Leung GJ, Rainsford KD, Kean WF (2014) Osteoarthritis of the hand I: aetiology and pathogenesis, risk factors, investigation and diagnosis. J Pharm Pharmacol 66(3):339-346

18. Lorea DP, Berthe JV, De Mey A et al (2002) The nerve supply of the trapeziometacarpal joint. JHS Eur 27B(3):232-237

19. Lorea P, Foucher G (2005) Joint denervation in hand surgery: a plea. Annales de chirurgie plastique esthétique 50:154-160

20. Pastrana MJ, Zaidenberg EE, Palumbo D, Cesca FJ, Zaidenberg CR (2018) Innervation of the proximal interphalangeal joint: an anatomical study. J Hand Surg Am 29:1e1-1e5
21. Pupon M, Duteille F, Cassagneau E et al (2004) Anatomical study of nerve supply of the trapeziometacarpal joint. Fifteen dissection. Rev Chir Orthop Reparatrice Appar Mot 90(4):346-352

22. Schultz R, Krishnamurthy S, Johnston A (1984) A gross anatomic and histologic study of the innervation of the proximal interphalangeal joint. J Hand Surg Am 95(5):669-674

23. Tuffaha SH, Quan A, Hashemi S, Parikh P, O'Brien-Coon DM, Broyles JM, Dellon AL, Lifchez SD (2019) Selective thumb carpometacarpal joint denervation for painful arthritis: clinical outcomes and cadaveric study. J Hand Surg Am 44(1):64.e1-64.e8

24. Wilder FV et al (2006) Joint-specific prevalence of osteoarthritis of the hand. Osteoarthr Cartil 14:953-957

25. Wilhelm A (1966) Die Gelenkdenervation und hire anatomischen Grundlagen. Hefte zurUnfallheilk 86:1-109

26. Zhang Y, Jordan JM (2010) Epidemiology of osteoarthritis. Clin Geriatr Med 26:355-369

Publisher's Note Springer Nature remains neutral with regard to jurisdictional claims in published maps and institutional affiliations. 